\title{
In diesem Ansatz steckt noch viel mehr drin!
}

\author{
Anmerkungen zum Tübinger Ansatz der Sprachförderung: \\ Fundament Anwendung im Theater
}

Maik Walter

\begin{abstract}
Theatercamps sind beliebt und fungieren in den letzten Jahren immer wieder auch als Labor für eine theoretisch fundierte Sprachförderung (Küppers \& Walter 2012). Häufig handelt es sich um drittmittelgeförderte Projekte und häufig weisen die Versprechen in den entsprechenden Anträgen ein hohes utopisches Potenzial auf. Diese Camps enden in der Regel mit einer Inszenierung und in den meisten Fällen liest man im Anschluss nicht mehr viel von den Projekten und deren Resultaten. Erfahrungen im theatralen Raum und Arbeitsergebnisse wissenschaftlich abgesichert zu erfassen und $\mathrm{zu}$ analysieren ist eine Herkulesaufgabe, der sich viele Dramapädagogen nicht stellen (können). Ob dies nun am fehlenden theoretischen Rüstzeug für eine angemessene Evaluierung liegt oder aber an der fehlenden Finanzierung einer entsprechenden Nachbereitungsphase, soll hier nicht erörtert werden. Trotzdem: Es gibt einige Ferienprojekte, die dies in vorbildlicher Weise tun und gerade die Sprachförderung kann dort mit Fug und Recht als Pionier der Evaluierung bezeichnet werden. Vielleicht auch weil der (fehlende) Zuwachs an sprachlichen Kompetenzen leichter festgestellt werden kann als mögliche Persönlichkeitsentwicklungen (Domkowsky \& Walter 2012), die für die Theaterarbeit immer wieder angeführt werden. Es gibt also Hoffnung und das ist die gute Nachricht, die in dem ersten hier zu besprechenden Buch weitergeben wird. Es handelt sich um einen Bericht eines Theatercamps. Die zweite zu besprechende Publikation ist ein linguistischer Baustein, der in dieses Projekt erfreulicherweise eingebaut wurde.
\end{abstract}

\section{Die Evaluation des Tübinger Theatercamps SStadt der Kinder"(Batzel/Bohl/Bryant 2013)}

Die ErziehungswissenschaftlerInnen Andrea Batzel und Thorsten Bohl sowie die Linguistin Doreen Bryant legen dort auf 164 Seiten die Evaluation des Tübinger Theatercamps SStadt der Kinder"vor. Nachdem das theoretische 
(Sprachförder-)Konzept (11-29) und die theoretischen (erziehungswissenschaftlichen) Grundlagen (S. 30-55) vorgestellt worden sind, werden die Forschungsfragen (56-59), die Methode (60-83) sowie die Ergebnisse (84-141) präsentiert und abschließend diskutiert (142-149).

Die Ausführungen zum theoretischen (Sprachförder-)Konzept bestehen im Kern aus der leicht veränderten Fassung des bereits in Scenario 1/2012 publizierten, online frei verfügbaren Aufsatzes zum Pfingstcamp $D a Z$ und Theater: Der dramapädagogische Ansatz zur Förderung der Bildungssprache. In den erziehungswissenschaftlichen Grundlagen werden die im Projekt evaluierten (nichtsprachlichen) Kompetenzen vorgestellt. Der pädagogisch-wissenschaftliche Duktus ist in diesem Kapitel streckenweise für DramapädagogInnen gewöhnungsbedürftig, umkreist aber viele auch für uns relevante Fragen: Was heißt genau sozial kompetent zu sein und wie kann eine solche Kompetenz evaluiert werden? Dieser Duktus mag auch daran liegen, dass diese Abschnitte zum Teil unter Mitarbeit von Studierenden entstanden sind. Die Ergebnisse einer meist mit viel Engagement und Energie angefertigten Abschlussarbeit werden nicht nur von den zu gutachtenden Hochschullehrenden zur Kenntnis genommen, sondern einer interessierten Öffentlichkeit zur Verfügung gestellt. Unter dem Aspekt einer in die aktuelle Forschung eingebundenen Lehre sowie der auf diese Weise praktizierten Nachwuchsförderung ist diese Entscheidung zwar als lobenswert hervorzuheben, erfordert aber auch Nachsicht von den Lesern.

Schauen wir uns den Bericht nun genauer an: Das Ziel der Evaluierung bestand darin, einerseits die Wirkungen des Pfingstcamps und andererseits die Meinungen der daran Beteiligten zu erfassen (56). Wirkungen konnten nur für den Sprachstand untersucht werden, da nur hier auch aussagekräftige Daten einer Kontrollgruppe existierten.

Sehr übersichtlich wird das Design der Evaluation dargestellt (60-63): vor und nach dem Camp wurden verschiedene Daten erhoben und ausgewertet. Die Evaluierung der sprachlichen Kompetenzen (SSprachwissenschaftliche Evaluation") erfolgte jeweils in diesen beiden Phasen in Form eines Aufsatzes, einer schriftlichen Bildbeschreibung und einer mündlichen frei elizitierten Produktion. Nach dem Camp wurden zudem die WorkshopleiterInnen in Fragebögen gebeten, die Leistungen der SchülerInnen einzuschätzen. Daneben wurden auch in einer "Überfachlichen Evaluation"weitere Daten wie Theatererfahrungen, soziale Kompetenzen oder Erwartungen erhoben und ausgewertet: Vor dem Camp mit Hilfe von Fragebögen für Eltern und SchülerInnen, nach dem Camp für SchülerInnen, Lehrende und Studierende. Abschließend wurden nach dem Camp auch Interviews geführt.

Von den mehr als 66 Kindern konnten 53 Fragebögen ausgewertet werden: Mehr als 80\% der befragten SchülerInnen wies einen Migrationshintergrund auf (64). Aus den Antworten der LehrerInnen in den Befragungen wurde geschlussfolgert, dass die LehrerInnen bei einer großen Anzahl der teilnehmenden Kinder keinen expliziten Sprachförderbedarf gesehen haben (123). Das verwundert nun erst einmal bei der Adressatengruppe des Camps "Kinder mit Migrationshintergrund aus Tübinger Schulen, die Förderbedarf in ihrer 
deutschen Sprachkompetenz aufwiesen"(17). Es steht zudem in deutlichem Widerspruch zu den sprachdidagnostischen Befunden der beteiligten Kinder. Und gerade diese Diskrepanz deutlich zu benennen, ist ein großes Verdienst des Berichts. Es macht nämlich deutlich, dass der Stand des gesicherten Wissens in der Forschung (hier die Diagnose des sprachlichen Förderbedarfs) in der Schulpraxis nicht ankommt. Über die Gründe einer solchen fehlenden diagnostische Kompetenz kann weiter spekuliert werden: Entweder gelingt es der Forschung nicht, ihre Ergebnisse für die LehrerInnen verständlich und für die Schulpraxis anwendbar aufzubereiten, oder es fehlen wissenschaftlich fundierte Lehrerfortbildungen, um diese Kompetenz auszubilden. Vielleicht handelt es sich auch nur um einen Tübinger Einzelfall, was zwar unwahrscheinlich ist, aber auch nicht ausgeschlossen werden kann. Die Diskrepanz in den Beurteilungen sollte nicht als Lehrerschelte missverstanden werden, denn Lehrende, die an einem solchen Projekt teilnehmen, zählen meist zu den engagierten KollegInnen, stecken zusätzliche Arbeitszeit in diese Projekte und tragen zum Erfolg bei.

Kommen wir zurück zum Bericht: Die Evaluationsinstrumente wurden für den Sprachstand vollständig angeführt. Dies entspricht dem wissenschaftlichen Standard und erlaubt es, die Verfahren für ähnliche Projekte anzupassen und dann in modifizierter $\mathrm{F}$ orm $\mathrm{z}$ u ü bernehmen. $\mathrm{D}$ ie $\mathrm{F}$ ragebögen $\mathrm{f}$ ür den allgemeinen Teil werden ebenfalls vorgestellt, wobei aber nur exemplarische Fragen, bzw. Aussagen angegeben werden. Dieses Vorgehen ist zwar in den Sozialwissenschaften weit verbreitet, als Dramapädagoge hätte ich in einem entsprechenden Buch erwartet, dass die Fragebögen vollständig zur Verfügung gestellt werden, um einerseits die Ergebnisse nachzuvollziehen und andererseits ein Werkzeug für künftige Camps zu haben. Gerade diese Möglichkeit hat man sich hier leider vergeben und von den allgemeinen Evaluationsinstrumenten kann man somit als DramapädagogIn mit Campambitionen nur eingeschränkt profitieren.

Wie sehen nun die Resultate der Evaluation aus? Die Ergebnisse der allgemeinen Befragung sind zum Teil ernüchternd: Bei den lern- und leistungsbezogenen Personenmerkmalen konnten keine signifikanten Veränderungen festgestellt werden. Die Lernmotivation beispielsweise ist in den Augen der GrundschülerInnen bereits vor dem Camp gut. In dem Alter werden die eigenen Kompetenzen jedoch häufig überschätzt und so wurden auch die LehrerInnen befragt. Diese wiederum sahen sich in vielen Fällen entweder nicht in der Lage, entsprechende Einschätzungen für die Kinder vorzunehmen oder bemerkten nach dem Camp bei der Mehrzahl der SchülerInnen keine Veränderungen (115-123).

Hervorzuheben sind die positiven Lerneffekte der dramagrammatischen Einheiten. Die Kinder des Camps wurden in Gruppen eingeteilt, die spezifische dramagrammatische Einheiten bearbeitet haben. Die Effekte dieser Arbeit - messbar in der Produktion von unterschiedlichen Nebensatztypen - sind beeindruckend: So ist der Zuwachs an Temporal- bzw. Kausalsätzen in den Sprachproben deutlich höher in den Gruppen, in denen diese Struktur 
fokussiert wurde (107-109). Die Ergebnisse legen eine sprachdidaktisch fundierte Theaterarbeit nahe. Dies lässt sich auch in einem anderen Bereich nachweisen, dem Ausdruck der räumlichen Beziehungen und damit kommen wir auch fast zur zweiten hier zu besprechenden Publikation, die gewissermaßen einen der linguistischen Bausteine des Sprachförderkonzepts darstellt. In der Evaluation wurden gezielt Strukturen eingefordert (elizitiert), in denen eine räumliche Beziehung ausgedrückt wird (Lokalisierungsausdrücke). In einer Bildbeschreibung wurden hierzu unterschiedliche Positionsmodi wie STEHEN oder HÄNGEN und Relationen wie IN oder AN getestet. Mit den entsprechenden Positionsverben, dem Gebrauch des Dativs sowie der einzelnen Lokalpräpositionen kann der Sprachgebrauch der Kinder beschrieben werden und ggf. auch deren Fortschritte im Lernprozess. Nachdem ein spezifischer Förderbedarf festgestellt wurde, und dementsprechend die Sprachförderung im Camp auch intensiviert wurde, zeigten sich nach dem Camp beträchtliche Zuwachsraten, und zwar zur Kontrollgruppe, aber auch zu den Teilgruppen, bei denen dieses Strukturen nicht im Fokus standen (113). Es zeigt sich, dass eine fundierte Sprachförderung durchaus Sinn macht und erfolgreich in ein Theaterprojekt integriert werden kann. Um solche Einheiten sinnvoll zu erarbeiten, muss man als DramagrammatikerIn wissen, wie das Deutsche in diesem Bereich strukturiert ist, wie sich der Spracherwerb gestaltet und mögliche Sprachfördermaßnahmen aussehen könnten. Darauf wird im zweiten Abschnitt zurück gekommen.

Die abschließende Diskussion des Berichts (142-149) fasst noch einmal die wesentlichen Ergebnisse der Evaluation prägnant zusammen, bevor Abbildungen, Tabellen, verwendete Literatur sowie Transkriptionsregeln angeführt werden.

Wahrscheinlich hätte dieser Bericht eine deutlich größere Leserschaft, wenn die Möglichkeiten der freien Publikation im Internet genutzt worden wären. Dies können universitäre oder aber auch institutionsübergreifende Plattformen sein. Ein Beispiel für den ersten Weg wäre die Universitätsbibliothek Tübingen, die Berichte kostenfrei als PDF mit einer dauerhaften Zitierangabe zur Verfügung stellt (http://tobias-lib.uni-tuebingen.de). Der zweite Weg wird beispielsweise von Stefan Müller und Martin Haspelmath mit der von ihnen angestoßenen Initiative Open Access in der Linguistik beschritten (http://hpsg.fu-berlin.de/OALI). Um auch in Zukunft wahrgenommen zu werden, sollte man diese Publikationsformen ernsthaft prüfen und, wenn nichts dagegen spricht, ihr den Vorzug gewähren.

\section{Lokalisierungsausdrücke im Erst- und Zweitspracherwerb (Bryant 2012)}

Das zweite hier zu besprechende Buch ist die überarbeitete Fassung einer Habilitationsschrift, die 2010 an der Universität Tübingen eingereicht wurde. In dieser Arbeit untersucht Doreen Bryant die bereits oben erwähnten 
Lokalisierungsausdrücke im Erst- und Zweitspracherwerb des Deutschen. Der Ausdruck des Raums ist ein immenses Lernproblem für Kinder, die Deutsch als Zweitsprache sprechen. Selbst Kinder, deren Erwerb des Deutschen als Zweitsprache sehr früh (im Alter von 2 bzw. 3 Jahren) beginnt, haben erhebliche Probleme, die Lokalisierungsausdrücke adäquat im Deutschen gebrauchen. Bryant begründet dies vor allem sprachtypologisch (52-54). Hierfür wird die entsprechende Lokalsystematik in den drei Sprachen Deutsch, Russisch und Türkisch entfaltet, die für die empirische Untersuchung relevant sind. Das Ziel dieser äußerst verdienstvollen Arbeit ist es, ein linguistisch und entwicklungspsychologisch abgesichertes Förderangebot für diesen klar umgrenzten Bereich zu begründen. Nichtlinguisten werden weite Teile dieses Buches nicht verstehen, wie das folgende Beispiel illustrieren soll.

$$
\text { leg-: } \lambda \mathrm{P} \lambda \mathrm{y} \lambda \underline{x}\left[\mathrm{CAUSE}\left(\mathrm{x}, \operatorname{BECOME}\left(\mathrm{P}(\mathrm{y}) \& \operatorname{MOD}_{\mathrm{LIEG}}(\mathrm{y})\right)\right)\right]
$$

Um am anspruchsvollen Diskurs teilzuhaben, sollte man so genannte Dekompositionsstrukturen wie den angeführten Lexikoneintrag des kausativen Positionsverbs legen (253) lesen können und wissen, dass rechtsperiphere Elemente nichts mit braunen Gedankengut zu tun haben, sondern vielmehr über die Anordnung sprachlicher Elemente in der syntaktischen Hierarchie Auskunft geben. Wer das aber kann oder sich auch mit etwas Zuversicht auf die 318 Seiten in den fünf Kapiteln einlässt, dem werden einige Lichter aufgehen, denn in diesem Buch steckt viel komprimiertes Wissen drin.

Beispielsweise wird schlüssig begründet, warum gerade doppelt markierte Strukturen (wie die im letzten Satz sowie im Titel angeführte) ausgesprochen wichtig für den DaZ-Erwerb sind: Neben der Präpositionalphrase in diesem Buch wird mit der Verbpartikel drin zusätzlich die räumliche Information angegeben. Umgangssprachliche Konstruktionen dieser Art erfüllen laut Bryant eine Stützfunktion beim Aufbau des Lokalisierungssystems (163), was sich bemerkenswerter Weise auch mit der hohen Frequenz dieser Strukturen im Input der Eltern nachweisen lässt. Dieser Input wird als einer der maßgeblichen Faktoren für einen erfolgreichen Erstspracherwerb angesehen und Bryant empfiehlt diese Konstruktionen in der Sprachförderung sogar noch zu verstärken. Dies ist originell und wird reine Praktiker erst einmal in Angst und Schrecken versetzen. Das Kind werde diese Redundanz jedoch bemerken und im Vorschulalter selbstständig zugunsten der lokalen Präpositionalphrasen reduzieren.

Als ein die Sprachförderung umfassendes Prinzip wird die typologisch ontogentisch kognitionspsychologisch motivierte Input-Anreicherung TOKIA ins Leben gerufen (12), die auch in den dramapädagogischen Kontext (280) eingebracht werden kann. Konkret bedeutet dies, dass der sprachliche Input in der Förderung mit ausgewählten sprachlichen Strukturen angereichert wird. Die Entscheidung, welche Strukturen dies sind, wird auf der Basis von sprachtypologischen und spracherwerbsbezogenen Erwägungen getroffen, wie beispielsweise im Falle der oben angeführten ungewohnten Doppelkonstruktion. Untermauert wird dieses Prinzip mit einer Reihe von empirischen Analysen. 
Bemerkenswert ist die Fülle von Daten, die hierfür zusammengetragen und ausgewertet wurden. Dazu stellte Bryant die bisherige Forschung dar, interpretierte die Daten zum Teil auch neu und erhob eigene Daten, deren Analyse der Gegenstand des experimentellen Kernkapitels (209-283) ist.

Das Buch liefert Instrumente für die Sprachstandsbestimmung, einen begründeten Vorschlag zur Progression sowie Möglichkeiten zur methodischen Umsetzung. All dies findet sich auch im Bericht des Theaterprojekts wieder und so zeigt Doreen Bryant sehr überzeugend, wie Sprachfördereinheiten wissenschaftlich abgesichert aufgebaut werden könnten. Interessant für die Dramapädagogik sind vor allem auch die Ausführungen und die Aufbereitung der Forschungsliteratur zur Verbindung von Spracherwerb, Sprachförderung und Körpererfahrung. Abschließend kann gesagt werden, dass es sich um eine der anspruchsvollsten und innovativsten Arbeiten handelt, die in den letzten Jahren im DaZ-Bereich geschrieben wurden. In der Dramapädagogik werden wir von den gewonnenen Erkenntnissen profitieren. Der Projektbericht über die Evaluation des Tübinger Theatercamps illustriert bereits, wie diese Reise aussehen kann.

Literatur -

Batzel, Andrea; Bohl, Thorsten; Bryant, Doreen (2013): Evaluation des Tübinger Theatercamps SStadt der Kinder". Ein Ferienprojekt zur Förderung von Sprache und sozialer Kompetenz. Baltmannsweiler: Schneider Verl. Hohengehren (Schul- und Unterrichtsforschung, 16)

Bryant, Doreen (2012): Lokalisierungsausdrücke im Erst- und Zweitspracherwerb. Typologische, ontogenetische und kognitionspsychologische Überlegungen zur Sprachförderung in DaZ. Tübingen, 2010. Baltmannsweiler: Schneider-Verl. Hohengehren (Thema Sprache - Wissenschaft für den Unterricht, 2)

Bryant, Doreen (2012): DaZ und Theater: Der dramapädagogische Ansatz zur Förderung der Bildungssprache. In: Scenario: Sprache - Kultur - Literatur VI (01), S. 28-58. Online verfügbar unter http://research.ucc.ie/scenario/2012/01/03bryant-2012-01-de.pdf

Domkowsky, Romi; Walter, Maik (2012): Was kann Theater? Ergebnisse empirischer Wirkungsforschung. In: Scenario VI/01, 104-126. Online verfügbar unter http://research.ucc.ie/scenario/2012/01/07domkowskywalter-2012-01-de.pdf

Küppers, Almut; Walter, Maik (2012): Theatermethoden auf dem Prüfstand der Forschung. Einführung in die Themenausgabe. In: Scenario VI/01, 1-9. Online verfügbar unter http://research.ucc.ie/scenario/2012/ 01/01-kuepperswalter-2012-01-de.pdf 